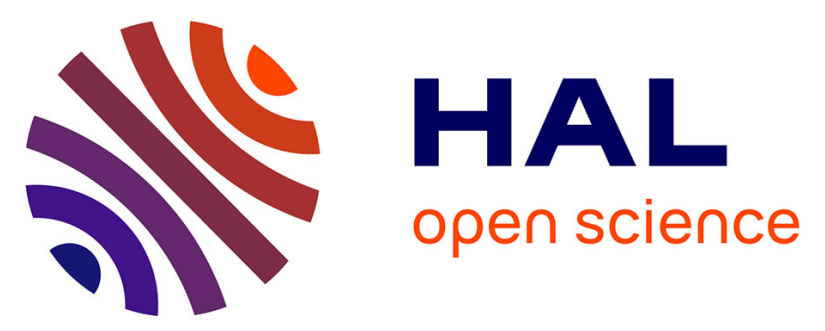

\title{
ÉTUDE DE LA TRANSITION DE FORME APLATIE $\rightarrow$ ALLONGÉE ENTRE 187Pt ET 185Pt
}

M. Deleplanque, C. Gerschel, M. Ishihara, N. Perrin, B. Ader, C. Bourgeois, J. Husson, P. Kilcher, J. Letessier

\section{To cite this version:}

M. Deleplanque, C. Gerschel, M. Ishihara, N. Perrin, B. Ader, et al.. ÉTUDE DE LA TRANSITION DE FORME APLATIE $\rightarrow$ ALLONGÉE ENTRE 187Pt ET 185Pt. Colloquium on Nuclear Surface, 1975, Dijon, France. pp.C5-97-C5-99, 10.1051/jphyscol:1975519 • jpa-00216374

\section{HAL Id: jpa-00216374 https://hal.science/jpa-00216374}

Submitted on 1 Jan 1975

HAL is a multi-disciplinary open access archive for the deposit and dissemination of scientific research documents, whether they are published or not. The documents may come from teaching and research institutions in France or abroad, or from public or private research centers.
L'archive ouverte pluridisciplinaire HAL, est destinée au dépôt et à la diffusion de documents scientifiques de niveau recherche, publiés ou non, émanant des établissements d'enseignement et de recherche français ou étrangers, des laboratoires publics ou privés. 


\title{
ÉTUdE DE LA TRANSITION DE FORME APLATIE $\rightarrow$ ALLONGÉE ENTRE ${ }^{187}$ Pt ET ${ }^{185} \mathbf{P t}$
}

\author{
(*) M. A. DELEPLANQUE, C. GERSCHEL, M. ISHIHARA, N. PERRIN et B. ADER
} (**) C. BOURGEOIS, J. P. HUSSON, P. KILCHER et Jocelyne LETESSIER

\author{
Institut de Physique Nucléaire, B.P. no 1, 91406 Orsay, France
}

\begin{abstract}
Résumé. - La mise en évidence par réaction $\left({ }^{16} \mathrm{O}, 5 \mathrm{n}\right)$ d'une bande découplée dans ${ }^{187} \mathrm{Pt}$ et d'une bande normale dans ${ }^{185} \mathrm{Pt}$ permet de prédire l'existence d'une transition oblate $\rightarrow$ prolate entre ces deux noyaux. De plus, les niveaux obtenus par radioactivité dans ${ }^{187} \mathrm{Pt}$ laissent supposer dans ce noyau la coexistence à basse énergie d'états de forme prolate.

Abstract. - By means of $\left({ }^{16} \mathrm{O}, 5 \mathrm{n}\right)$ reaction, we have given evidence for a decoupled band in ${ }^{187} \mathrm{Pt}$ and a strongly coupled one in ${ }^{185} \mathrm{Pt}$. This can be explained by a transition between oblate and prolate shapes. Moreover, in ${ }^{187} \mathrm{Pt}$, levels observed by radioactivity studies allow to predict the coexistence of prolate shape at low energy.
\end{abstract}

1. Introduction. - Des calculs effectués par Baranger et Kumar [1] sur les noyaux pair-pair de platine $(Z=78)$ et d'osmium $(Z=76)$ avaient permis de prédire l'existence de déformations aplaties (oblate) dans cette région. Dans les platines pair-pair, le groupe français d'Isolde (CERN) a proposé [2] une transition entre la forme oblate et la forme prolate (allongée) entre $N=110$ et 108, en accord avec les prédictions de Kumar [3]. Dans les noyaux de mercure, la transition oblate $\rightarrow$ prolate se produit de façon décalée pour les noyaux impairs et pour les noyaux pair-pair dans leurs états fondamentaux. En effet, la transition est observée entre ${ }^{187} \mathrm{Hg}(N=107)$ et ${ }^{185} \mathrm{Hg}(N=105)$ [4] alors que ${ }^{184} \mathrm{Hg}(N=104)$ est encore oblate dans son état fondamental $[5,6,7]$ L'étude de ${ }^{187} \mathrm{Pt}$ et ${ }^{185} \mathrm{Pt}$ peut donc permettre d'apporter des éléments nouveaux quant aux formes des noyaux de cette région.

2. Méthodes expérimentales. - Les expériences ont été entreprises simultanément par réaction

$$
{ }^{174,176} \mathrm{Yb}\left({ }^{16} \mathrm{O}, 5 \mathrm{n}\right){ }^{185,187} \mathrm{Pt}
$$

au CEV d'Orsay [8] et par décroissance radioactive de ${ }^{187} \mathrm{Au}\left(T_{1 / 2}=8 \mathrm{~min}\right.$.) à l'aide du séparateur Isocèle en ligne sur le synchrocyclotron d'Orsay [9]. Les mesures effectuées dans la réaction $\left({ }^{16} \mathrm{O}, 5 \mathrm{n}\right)$ sont présentées dans la référence [8]. Les études de radioactivité sont réalisées dans les conditions décrites dans la référence [10], à l'exception de l'usage d'une cible de platine d'un type nouveau (alliage platinebore fondu) [9].

(*) Réactions (IL, $\mathrm{xn}$ ).

$\left({ }^{* *}\right)$ Radioactivité en ligne.
3. Résultats expérimentaux. - Les schémas de niveaux partiels obtenus par ces deux méthodes expérimentales sont présentés sur les figures 1 et 2 .

3.1 Radionctivité (Fig. 1). - Pt 187 : on peut prédire un spin $3 / 2^{-}$ou $5 / 2^{-}$pour l'état fondamental de ${ }^{187} \mathrm{Pt}$ connaissant sa décroissance vers les états de ${ }^{187}$ Ir [11]. Par ailleurs, l'alimentation importante des états à 1,341 et $1,383 \mathrm{MeV}$ à partir de l'état $1 / 2^{+}$[10] de ${ }^{187} \mathrm{Au}$ permet de leur attribuer un spin $1 / 2^{+}$ou $3 / 2^{+}$. L'ensemble du schéma de niveaux proposé permet une interprétation cohérente des résultats expérimentaux. En particulier la présence des deux états isomériques situés à 242,1 et $260,0 \mathrm{keV}$ a facilité la construction du schéma.

3.2 Ions Lourds (FIg. 2). - Pt 187 : la caractéristique principale de ce schéma est l'existence d'une bande de transitions de caractère stretched $E 2$ dont les énergies sont proches de celles de la bande fondamentale de ${ }^{188} \mathrm{Pt}$. Aucun recouvrement n'est observé avec les résultats de radioactivité.

Pt 185 : dans ce cas, la seule bande observée n'est pas découplée mais formée de transitions $M 1+\mathrm{E} 2$ avec transitions parallèles $\mathrm{E} 2$. La transition de $94,8 \mathrm{keV}$ proposée comme piédestal du schéma vue son intensité [8] n'a pas été incluse dans cette bande sur la figure 2 car la transition de $211,8 \mathrm{keV}$ qui doit servir de transition parallèle à la cascade $94,8-117,4 \mathrm{keV}$ est très faible. Néanmoins, cette bande, si l'on inclut la transition de $94,8 \mathrm{keV}$, ressemble très fortement à la bande $9 / 2^{+}[624]$ observé dans l'isotone $(\mathrm{N}=107)$ ${ }^{183}$ Os [12] (voir Fig. 3).

4. Discussion. - L'observation d'une bande découplée dans ${ }^{187} \mathrm{Pt}$ implique [13] l'existence d'orbitales de $j$ élevé au voisinage du niveau de Fermi. On se trouve ici en fin de couches h 9/2 et i 13/2. Pour avoir 


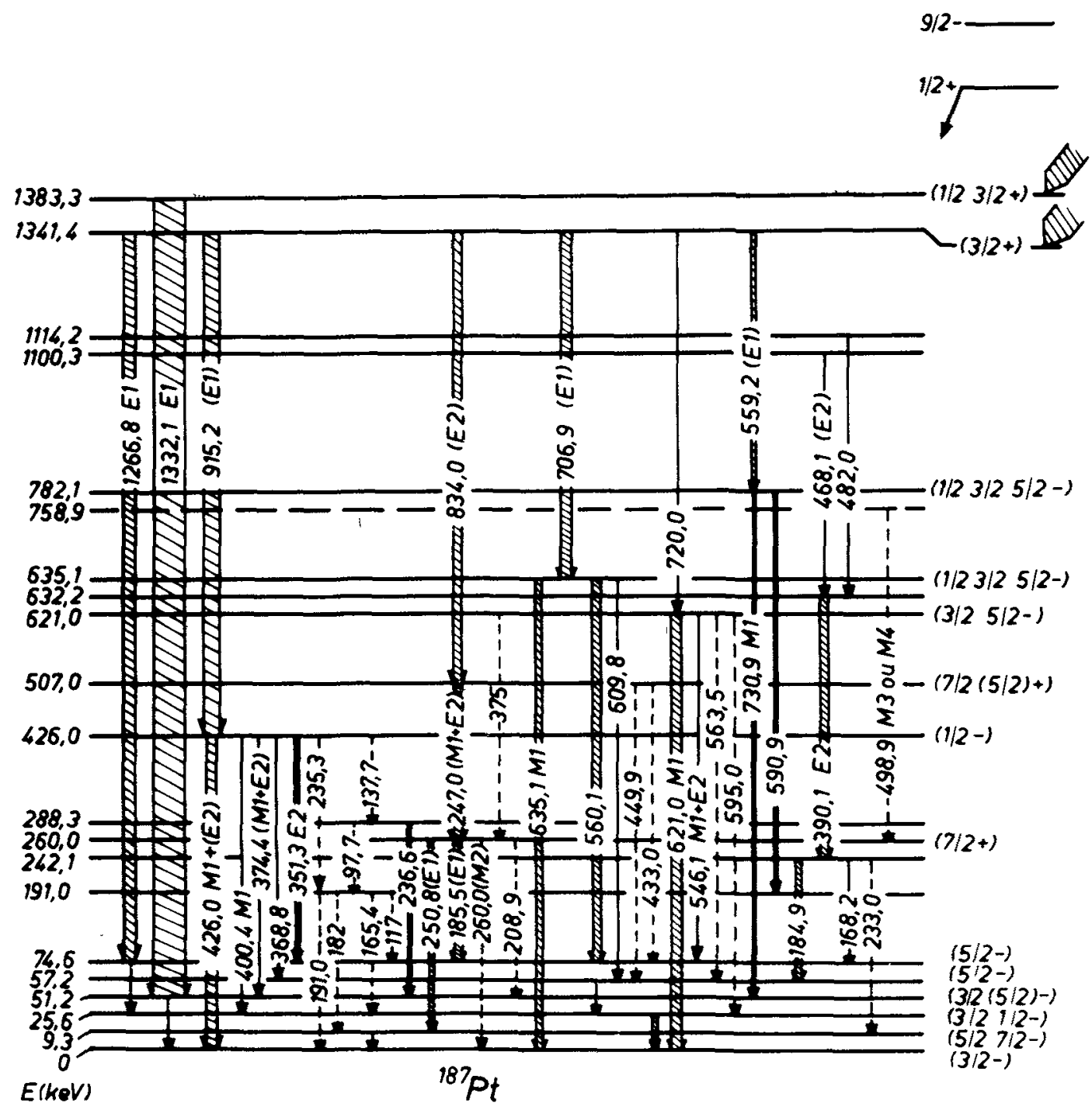

Fig. 1. - Schéma de niveaux partiel de ${ }^{187} \mathrm{Pt}$ obtenu par radioactivité. Les transitions de $9,3,25,6,31,7,49,0$ et $51,2 \mathrm{keV}$ sont représentées par un trait fin, leurs intensités totales n'ayant pas encore été évaluées exactement.

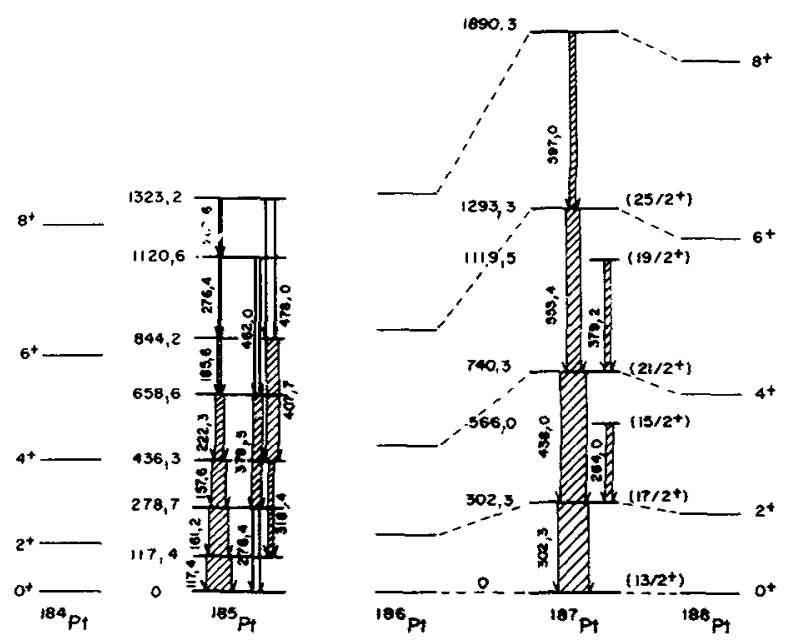

FIG. 2. - Schéma de niveaux partiel des ${ }^{185} \mathrm{Pt}$ et ${ }^{187} \mathrm{Pt}$ obtenus par réaction $\left({ }^{16} \mathrm{O}, 5 \mathrm{n}\right)$.

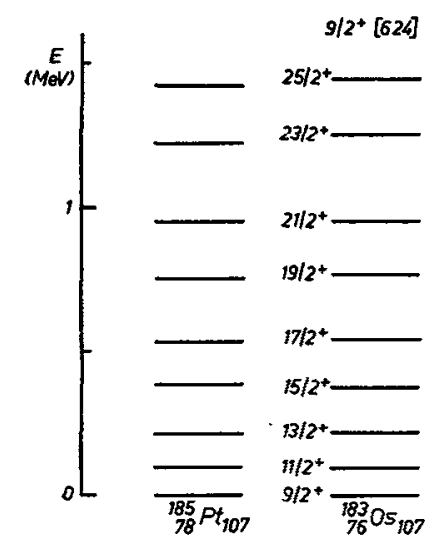

FIG. 3. - Comparaison entre la bande excitée dans ${ }^{185} \mathrm{Pt}$ et la bande $9 / 2^{+}[624]$ de ${ }^{183}$ Os. 
une bande découplée, il faut donc que le noyau ait une déformation oblate. L'existence dans les platines impairs [14] d'états isomériques $13 / 2^{+}$dont l'énergie décroit de $A=199(425 \mathrm{keV})$ à $A=193(149 \mathrm{keV})$ indique que le spin du niveau de base est $13 / 2^{+}$plutôt que $9 / 2^{-}$. Ceci est d'ailleurs cohérent avec la systématique proposée [15] dans les platines de masse $A \geqslant 187$.

On peut penser que cet état $13 / 2^{+}$est l'état isomérique [16] dont nous avons mesuré la période $\left(T_{1 / 2}=250 \pm 50 \mu \mathrm{s}\right)$ par la décroissance d'une raie de $120 \mathrm{keV}$ et de rayons $\mathrm{X}_{\mathrm{K}}\left(\mathrm{I}_{\mathbf{X K}} / \mathrm{I}_{120} \simeq 7\right)$. Ces résultats expérimentaux sont compatibles avec une transition M 2 de $120 \mathrm{keV}$ qui pourrait être une transition $13 / 2^{+} \rightarrow 9 / 2^{-}$. Néanmoins quels que soient l'énergie et le spin de l'état fondamental de la bande observée, son caractère découplé implique une forme oblate pour ces états.

Dans ${ }^{185} \mathrm{Pt}$, il est intéressant de noter que la situation a changé par rapport à ${ }^{187} \mathrm{Pt}$ puisque, ici, la bande la plus alimentée n'est pas découplée. Nous n'avons pas pu fixer les spins dans cette bande mais la grande similitude avec ${ }^{183} \mathrm{Os}$ (Fig. 3) permet de penser qu'il s'agit de la bande $9 / 2^{+}$[624]. On aurait donc des états bien déformés et prolate.

Dans ${ }^{187} \mathrm{Pt}$, les niveaux mis en évidence en radioactivité ou par réaction sont différents. Ceci tient au fait que le spin de ${ }^{187} \mathrm{Au}$ est $1 / 2^{+}$et alimente préfé- rentiellement les états de bas spin de ${ }^{187} \mathrm{Pt}$. Les résultats de radioactivité sont encore préliminaires mais on peut néanmoins insister sur les points suivants :

- La forte densité de niveaux à basse énergie suggère pour ces états une déformation plus grande que celle des états observés par ions lourds. De plus, dans l'hypothèse faite où l'état de $260,0 \mathrm{keV}$ a un spin $7 / 2^{+}$, on est obligé de faire appel à une déformation prolate pour expliquer sa présence à si basse énergie.

- Les états fortement alimentés à 1,341 et $1,383 \mathrm{MeV}$ sont comparables à l'état de $1,16 \mathrm{MeV}$ de ${ }^{189} \mathrm{Pt}$ [17] interprété par l'analyse des $\beta$ strength function.

- L'observation d'une transition de $498,9 \mathrm{keV}$ (M 3 ou M 4) devrait favoriser l'existence de niveaux de haut spin. Dans ce cas, il est surprenant de ne pas observer par radioactivité le niveau $13 / 2^{+}$.

L'analyse des bandes observées dans ${ }^{187} \mathrm{Pt}$ et ${ }^{185} \mathrm{Pt}$ semble donc bien signer l'existence de déformations différentes dans ces 2 noyaux et en accord avec celles observées dans les platines pair-pair voisins. De plus, les résultats de radioactivité semblent indiquer que les deux déformations peuvent coexister dans ${ }^{187} \mathrm{Pt}$.

Remerciements. - Nous remercions toute l'équipe d'Isocèle et plus particulièrement MM. J. Obert et J. C. Putaux qui ont résolu tous les problèmes inhérents à la cible de platine.

\section{Bibliographie}

[1] Baranger, M. et Kumar, K., Phys. Rev. Lett. 17 (1966) 1146.

[2] Finger, M. et al., Nucl. Phys. A 188 (1972) 369.

[3] Kumar, K., Int. Conf. on the Properties of Nuclei far from the region of beta stability, Leysin-CERN-Report 70-30 (1970) p. 779.

[4] Bonn, J., Huber, G., Kluge, H.-J., KöpF, U., Kugler, L., Otten, E. W., Phys. Lett. 38B (1972) 308.

[5] Proetex, D., Diamond, R. M., Kienle, P., Leigh, J. R., Maier, K. M. et Stephens, F. S., Phys. Rev. Lett. 31 (1973) 896.

[6] Proetel, D., Stephens, F. S., Diamond, R. M., Int. Conf. on Reactions between Complex Nuclei, Nashville, USA (1974) 162.

[7] Rud, N., Ward, D., Andrews, H. R., Graham, R. L. et GeIGER, J. S., Phys. Rev. Lett. 31 (1973) 1421.
[8] Deleplanque, M. A., Gerschel, C., Ishithara, M., Perrin, N. et Ader, B., C. R. Hebd. Séan. Acad. Sci. 280 (1975) 515.

[9] The Orsay on-line separator : Isocèle (à paraître).

[10] Deleplanque, M. A. et al., J. Physique Lett. 36 (1975) L 205.

[11] SebIlle-SCHücK, C. et al., Nucl. Phys. A 212 (1973) 45.

[12] Lindelad, T., Bethoux, R., Price, R. H. et Kleinheinz, P., Nucl. Phys. A 217 (1973) 459.

[13] Stephens, F. S., Diamond, R. M., Leigh, J. R., Kammuri, T. et NAKaI, K., Phys. Rev. Lett. 29 (1972) 438.

[14] Nuclear Data Sheets (Academic Press, Inc.) 1973.

[15] PitParinen, M. et al., Phys. Rev. Lett. 34 (1975) 1110.

[16] Ader, B., Deleplanque, M. A., Gerschel, C., Leblanc, M. et PERrin, N. (à paraître).

[17] Jastrzebski, J., Kilcher, P. et PARIs, P., J. Physique 34 (1973) 755. 